\title{
AN EXPLANATION OF THE POLAR MOTION BY A RIGID CORE-MANTLE MODEL
}

\author{
YOSHIO KUBO \\ Hydrographic Department, Tokyo, Japan
}

\begin{abstract}
The Earth with a rigid core and a rigid mantle is considered and a recovering force is assumed between the constituents.

The equations of motion in canonical form are derived in terms of the Andoyer's variables. Applying the perturbation theory, the phenomenon of the polar motion is investigated.

The polar motion obtained with this model is a circular motion of the rotational axis on the mantle's surface with its radius gradually changing.
\end{abstract}

The Earth is assumed to consist of a rigid core and a rigid mantle. It is further assumed that there exists an equilibrium configuration between the core and the mantle and, when the two constituents deviate from this state, a torque proportional to the deviation occurs; i.e. if we denote the deviation by $\Delta \alpha$ and the resultant torque by $N$,

$$
N=\sigma \cdot \Delta \alpha .
$$

The potential energy is given by

$$
U=\frac{1}{2} \sigma(\Delta \alpha)^{2} .
$$

In the equilibrium state the principal axes of inertia of the core and the mantle need not necessarily coincide with each other. Let the core have principal moments of inertia $A_{1}, B_{1}$ and $C_{1}$, and the mantle $A_{2}, B_{2}$ and $C_{2}$ (But we confine our discussion to the case in which $A_{1}=B_{1}$ and $A_{2}=B_{2}$ ); then the $C_{2}$-axis may be assumed to deviate by an angle $\gamma$ from the $C_{1}$-axis towards the $A_{1}$-axis in the state of equilibrium (Figure 1). With this assumption the potential is approximately expressed as

$$
\begin{aligned}
U=\frac{1}{2} \sigma\left\{3-\left(\cos \overparen{A_{1} A_{2}}+\cos \overparen{C_{1} C_{2}}\right) \cos \gamma\right. & -\cos \overparen{B_{1} B_{2}}- \\
& \left.-\left(\cos \overparen{A_{1} C_{2}}-\cos \overparen{A_{2} C_{1}}\right) \sin \gamma\right\} .
\end{aligned}
$$

Since $\gamma$ is considered small, we can write $\cos \gamma=1$ and $\sin \gamma=\gamma$.

We write the Hamiltonian of the system in terms of Andoyer's canonical variables. They are $\left(L_{1}, G_{1}, H_{1}, l_{1}, g_{1}, h_{1}\right)$ for the core and $\left(L_{2}, G_{2}, H_{2}, l_{2}, g_{2}, h_{2}\right)$ for the mantle. The Hamiltonian is written

$$
F=\frac{1}{2 A_{1}}\left(L_{1}^{2}-G_{1}^{2}\right)-\frac{1}{2 C_{1}} L_{1}^{2}+\frac{1}{2 A_{2}}\left(L_{2}^{2}-G_{2}^{2}\right)-\frac{1}{2 C_{2}} L_{2}^{2}-U-V,
$$

where $V$ is the potential of the Moon and the Sun. 


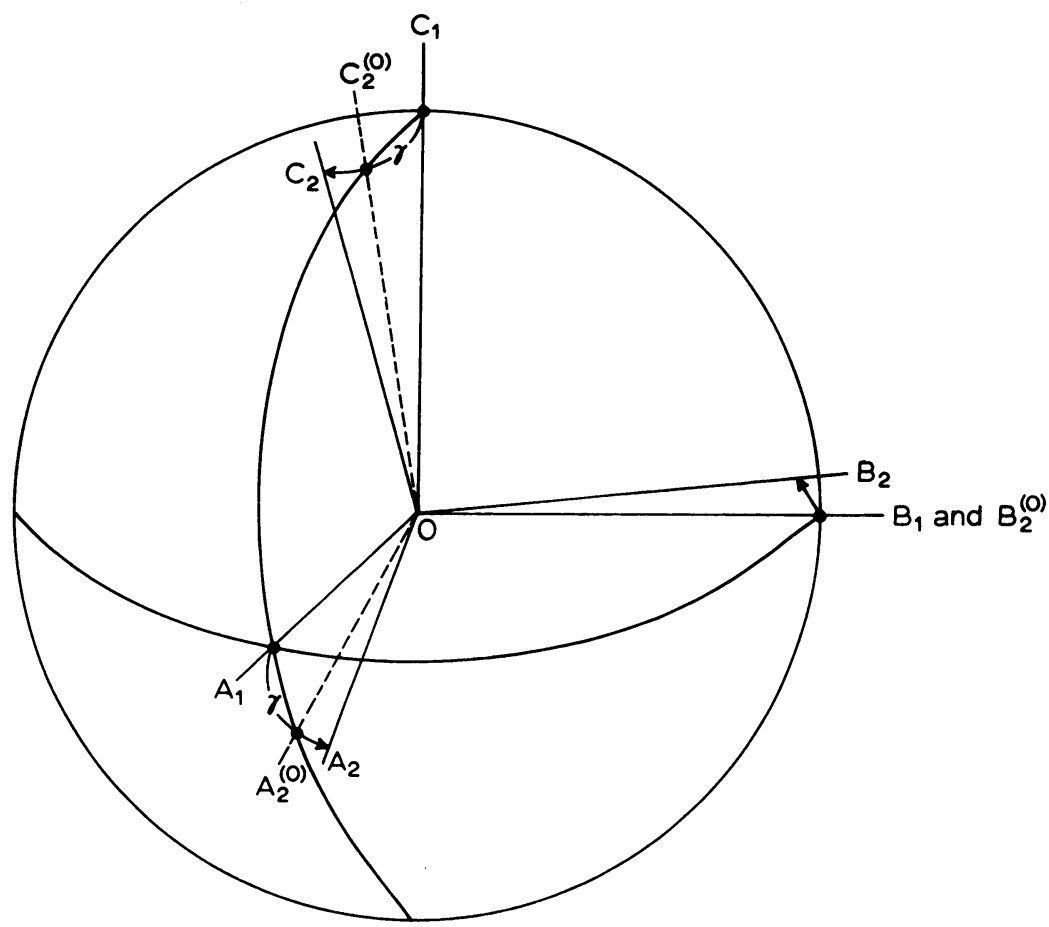

Fig. 1. Configuration of the core and the mantle. Suffix 1: core; suffix 2: mantle. $A_{2}{ }^{(0)}, B_{2}{ }^{(0)}, C_{2}{ }^{(0)}$ : configuration in the equilibrium state. $\overparen{A_{1} A_{2}}=\left\langle A_{1} \circ A_{2}\right.$ etc.

The diurnal terms are first removed by the method of canonical transformation. Hence the new Hamiltonian is

$$
\begin{aligned}
F^{*}= & \frac{1}{2 A_{1}}\left(L_{1}^{2}-G_{1}^{2}\right)-\frac{1}{2 C_{1}} L_{1}^{2}+\frac{1}{2 A_{1}}\left(L_{2}^{2}-G_{2}^{2}\right)-\frac{1}{2 C_{2}} L_{2}^{2}-V^{*}+ \\
& +\frac{\sigma}{4}\left[\left(\cos h^{\prime}+\cos h^{\prime} \cos r_{1} \cos r_{2}+\sin r_{1} \sin r_{2}\right) \times\right. \\
& \times\left\{\frac{1}{2}\left(1+\cos s_{1}\right)\left(1+\cos s_{2}\right) \cos \left(l^{\prime}+g^{\prime}\right)+\sin s_{1} \sin s_{2} \cos g^{\prime}\right\}+ \\
& +2\left(\cos h^{\prime} \sin r_{1} \sin r_{2}+\cos r_{1} \cos r_{2}\right) \times \\
& \times\left(\sin s_{1} \sin s_{2} \cos l^{\prime}+\cos s_{1} \cos s_{2}\right)+ \\
& +4 \gamma\left(\sin s_{1} \sin \frac{l_{1}+l_{2}-g^{\prime}}{2} \cos \frac{l^{\prime}+g^{\prime}}{2}-\right. \\
& \left.\left.-\sin s_{2} \sin \frac{l_{1}+l_{2}+g^{\prime} \cos +g^{\prime}}{2}\right)\right]
\end{aligned}
$$

where $l^{\prime}=l_{1}-l_{2}, g^{\prime}=g_{1}-g_{2}, h^{\prime}=h_{1}-h_{2}, \cos r_{i}=H_{i} / G_{i}$ and $\cos s_{i}=L_{i} / G_{i}(i=1,2)$. 
We now introduce new pairs of conjugate variables $x_{i}=L_{i}-G_{i}$ and $y_{i}=l_{i}(i=1,2)$, which represent the polar motion most directly. The other variables are $G_{i}, l_{i}+g_{i}, H_{i}$ and $h_{i}(i=1,2)$. By the term 'polar motion', the motion of each rotational axis relative to each corresponding pole of the figure is meant. It should be noticed, however, that the observable polar motion is that for the mantle. Then it is seen that the behaviors of $x_{i}$ and $y_{i}$ are almost independent of those of the other variables. That is, the polar motions hardly depend on the motions of the angular momentum vectors. (These vectors can be considered as practically coincident with the respective rotational axes.) It is also seen that the external forces have no considerable effect on the $x_{i}$ and $y_{i}$. The sum of the two angular momentum vectors moves in the same way as in the case of a rigid earth under the influence of these forces.

The polar motions are thus given by the equations

$$
\begin{aligned}
f= & \left(\frac{G_{1}}{A_{1}}-\frac{G_{1}}{C_{1}}\right) x_{1}+\left(\begin{array}{l}
G_{2} \\
A_{2}
\end{array}-\frac{G_{2}}{C_{2}}\right) x_{2} \\
& +\frac{\sigma}{4}\left[\frac{1}{2}\left(1+\cos s_{1}\right)\left(1+\cos s_{2}\right)+\sin s_{1} \sin s_{2} \cos \left(y_{1}-y_{2}\right)+\right. \\
& \left.+4 \gamma\left(\sin s_{1} \sin y_{1}-\sin s_{2} \sin y_{2}\right)\right], \\
\dot{x}_{i}= & \frac{\partial f}{\partial y_{i}}, \quad \dot{y}_{i}=-\frac{\partial f}{\partial x_{i}} \quad(i=1,2) .
\end{aligned}
$$

Solving these equations by numerical integration, we obtain some general results.

(i) The rotational axes of the core and the mantle make circular motions relative to the respective coordinate axes fixed in the core and the mantle.

(ii) The periods of the circular motions are different from 305 days.

(iii) The radii of the circles in the core and the mantle both suffer a gradual periodic change.

(iv) The period of this change depends on the values for $\sigma,\left(C_{1}-A_{1}\right) / C_{1}$ and $\left(C_{2}-A_{2}\right) / C_{2}$.

(v) The amplitude is determined by the value of $\sigma$ and the initial conditions.

(vi) The effect of $\gamma$ is rather curious. It makes the centers of the circular motions deviate from the respective poles of the figure. The aspect of the motion, however, is quite the same as when $\gamma=0$.

\section{Reference}

Andoyer, H.: 1926, Mécanique Céleste, Gauthier-Villars, Paris, T. II, p. 357. 